\title{
Hepatoid adenocarcinoma of the stomach: A case report and review of the literature
}

\author{
RU ZHOU ${ }^{1 *}$, YANTAO CAI $^{2 *}$, YI YANG $^{2}$, JIANBIN XIANG $^{2}$ and ZONGYOU CHEN ${ }^{2}$ \\ ${ }^{1}$ Department of General Surgery, Luwan Branch, Ruijin Hospital, Shanghai Jiaotong University School of Medicine, \\ Shanghai 200020; ${ }^{2}$ Department of General Surgery, Huashan Hospital, Fudan University, Shanghai 200040, P.R. China
}

Received December 22, 2013; Accepted October 16, 2014

DOI: $10.3892 / \mathrm{ol} .2015 .2979$

\begin{abstract}
Hepatoid adenocarcinoma is a rare tumor, associated with an extremely poor prognosis. In the present report, a 72-year-old female presented to Huashan Hospital (Shanghai, China) with upper abdominal discomfort and, following endoscopy, an ulcerated lesion was observed in the stomach. A computed tomography scan revealed a mass in the antrum of the stomach and the $\alpha$-fetoprotein level was normal. Sections of the mass exhibited malignant cells arranged in a solid to trabecular pattern; the cells were polygonal shaped with well-defined cytoplasmic borders. Immunohistochemical analysis was performed and the cells were positive for Hep1, CK, CK8, CK18 and P53. The histological features together with the immunohistochemical findings were diagnostic of a hepatoid adenocarcinoma of the stomach. Immunohistochemical studies may aid in the identification of the characteristic features and prevent the misdiagnosis of this tumor.
\end{abstract}

\section{Introduction}

Hepatoid adenocarcinoma of the stomach (HAS) is a particular type of extrahepatic adenocarcinoma, presenting with morphological characteristics identical to those of hepatocellular carcinoma (HCC). A case of $\alpha$-fetoprotein-producing gastric adenocarcinoma was reported by Ishikura et al (1) in 1985. The authors suggested the name of HAS due to the ability of the tumor cells to produce $\alpha$-fetoprotein (AFP), which is characteristic of hepatoid adenocarcinoma cells (1). HAS is reported to occur with more frequent lymph node and liver metastasis and exhibits a poorer prognosis than common gastric cancer (CGC) (2). HAS is rare (with a worldwide incidence of $0.3-1 \%$ )

Correspondence to: Professor Zongyou Chen, Department of General Surgery, Huashan Hospital, Fudan University, 12 Wulumuqi Zhong Lu, Shanghai 200040, P.R. China E-mail: zhou_richard@126.com

*Contributed equally

Key words: hepatoid adenocarcinoma, immunohistochemistry, gastric adenocarcinoma of all kinds of gastric cancer (2-4), the sypmtoms are similar to those of normal gastric cancer (abdominal discomfort, fullness of anorexia, epigastric pain, voimiting and weight loss), surgery is the usual treatment option (4-7). The literature review presented in this study was conducted to contribute towards the improvement of the diagnosis and treatment of HAS (3). We report a case of a 72-year-old female patient who sufferd upper abdominal discomfort. Endoscopical and radiological examination revealed a neoplasm in the stomach, however serum $\alpha$-fetoprotein levels were normal. Written informed consent was obtained from the patient's family.

\section{Case report}

Case presentation. A 72-year-old Chinese female presented to Huashan Hospital (Shanghai, China) complaining of upper abdominal discomfort for the previous two months. The patient was admitted to the General Surgery Department of Huashan Hospital on October 8th, 2011. The results of the physical examinations were unremarkable. The past medical history revealed nothing significant; the patient had not previously undergone any surgery and there was no family history of cancer. The laboratory investigation revealed normal blood levels following routine tests, and liver and kidney function were normal. The white blood cell count was $6.8 \times 10^{9} / 1$ (normal range, $4.5-11 \times 10^{9} / 1$ ), the hematocrit level was $120 \mathrm{~g} / 1$ (normal range, $110-150 \mathrm{~g} / \mathrm{l})$, the red blood cell count was $3.93 \times 10^{12} / 1$ (normal range, $3.5-5.0 \times 10^{12} / 1$ ), alanine aminotransferase levels were $11 \mathrm{U} / 1$ (normal range, 0-50 U/1), aspartate aminotransferase level was $18 \mathrm{U} / 1$ (normal range, 0-30 U/1), the bilirubin level was $12 \mu \mathrm{mol} / 1$ (normal range, 3.4-20.4 $\mu \mathrm{mol} / \mathrm{l}$ ), the serum creatinine level was $87 \mu \mathrm{mol} / 1$ (normal range, $50-130 \mu \mathrm{mol} / \mathrm{l}$ ) and the blood urea nitrogen level was $5.3 \mathrm{mmol} / 1$ (normal range, $2.5-7.0 \mathrm{mmol} / \mathrm{l}$ ) In addition, the AFP level was $4.93 \mu \mathrm{g} / \mathrm{l}$ (normal value, $<10 \mu \mathrm{g} / \mathrm{l}$ ), carcinoembryonic antigen (CEA) level $0.69 \mu \mathrm{g} / \mathrm{l}$ (normal range, $0-10 \mu \mathrm{g} / \mathrm{l}$ ), a CA125 level of $30.55 \mathrm{U} /$ $\mathrm{ml}$ (normal range, $0-35 \mathrm{U} / \mathrm{ml}$ ) and the CA724 levels of $2.5 \mathrm{U} / \mathrm{ml}$ (normal range, 0-8.2 U/ml), were all within the normal ranges. Furthermore, the hepatitis $\mathrm{B}$ and $\mathrm{C}$ panels were negative. A gastroduodenoscopy revealed a large gastric antrum ulcer and, subsequently, irregular and hyperchromatic nuclei were observed in the carcinoma cells, which was diagnosed as a poorly differentiated adenocarcinoma. Computed tomography was performed and mural thickening of the gastric antrum 

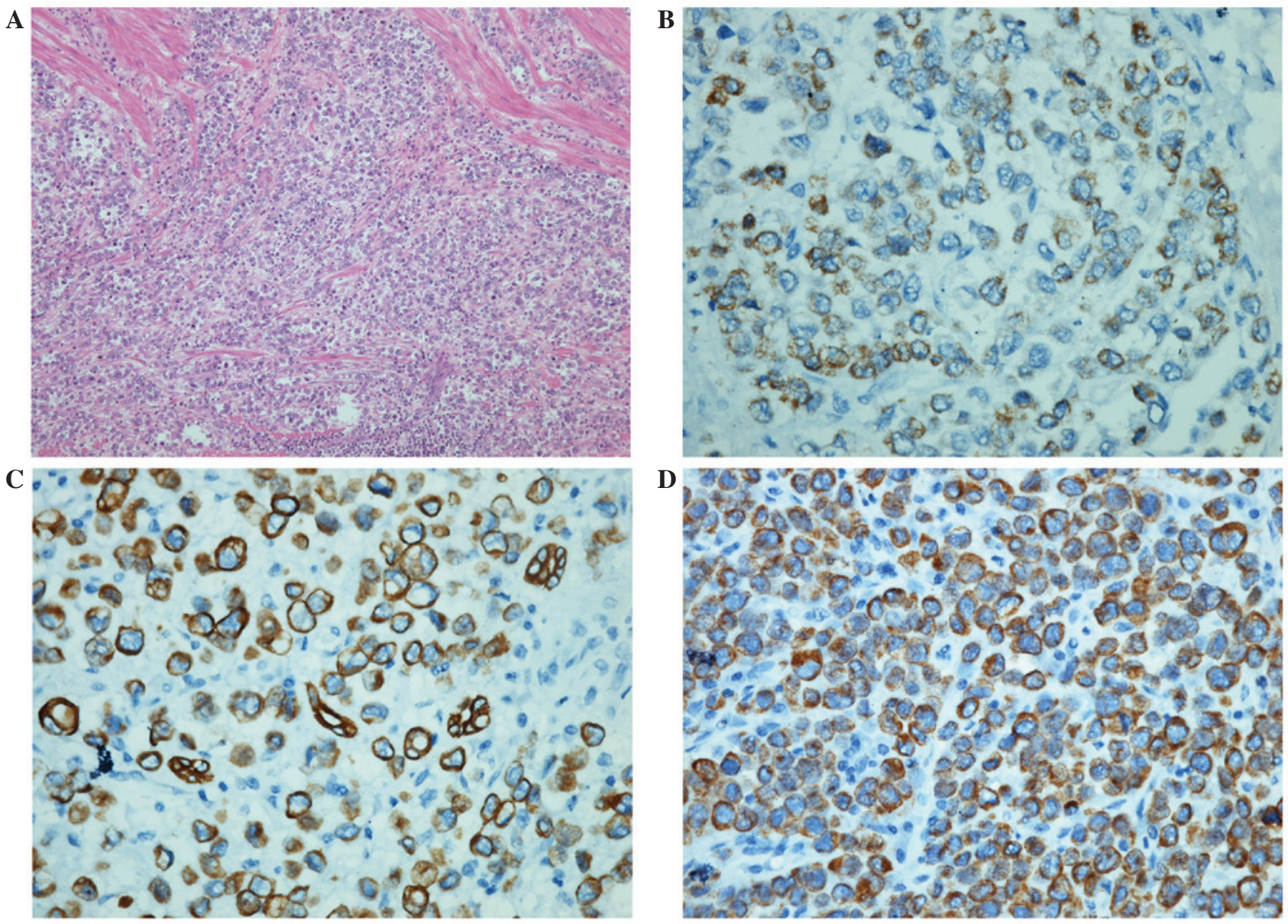

Figure 1. (A) Hematoxylin and eosin staining (magnification, x100). Cancer cells were polygonal, with an abundant eosinophilic cytoplasm, the nuclei were centered, large and irregular and the nucleolus was clearly visible. Positive staining was observed for (B) Hep1, (C) CK8 and (D) CK18 (magnification, $\mathrm{x} 400$ ).

was observed, as well as a gastric mass, measuring $10 \times 5 \mathrm{~cm}$ in diameter. In addition, massive lymph node swelling around the greater and lesser curvature of the stomach, head of pancreas, portal and splenic vein was evident.

Treatment. The patient underwent a distal gastrectomy plus resection of the small intestine with tumor debulking and subsequent chemotherapy (150 mg oxaliplatin with $3.5 \mathrm{~g}$ fluorouracil and $300 \mathrm{mg}$ folinic acid, six cycles). For the first two years after surgery, the patient was followed-up every three-four months, then every six months thereafter. The patient was without relapse at the time of writing.

Pathological analysis. The pathological analysis was conducted on the resected specimen, which consisted of stomach tissue, measuring $22 \mathrm{~cm}$ in the greater gastric curvature and $15 \mathrm{~cm}$ in the lesser curvature. The serosal surface showed an ill-defined, hemorrhagic, necrotic mass, measuring $10.5 \times 6 \times 1.5 \mathrm{~cm}$, which invaded into the whole gastric wall. Sectioning of the mass revealed a gray-white, homogeneous cut surface with scattered areas of hemorrhage and necrosis. Sections of the mass exhibited malignant cells arranged in a solid to trabecular pattern (Fig. 1A). The cells were polygonal shaped with well-defined cytoplasmic borders. The cytoplasm was clear to eosinophilic and a number of the cells exhibited centrally located hyperchromatic nuclei. Immunohistochemical analysis was performed to further characterize the tumor. The tumor cells were immunohistochemically positive for Hep1 (Fig. 1B), CK, CK8 (Fig. 1C), CK18 (Fig. 1D), P53 and Ki67 (50\%+), and negative for Vim, LCA, ChroA, Syn and CerbB-2.

\section{Discussion}

HAS originates from gastric mucosa, exhibiting morphological features of CGC and hepatoid adenocarcinoma, and has been classified as a rare subtype of gastric malignant tumors (4). According to recent studies, the incidence among gastric cancer cases was $1.5-15 \%(5,6)$. The mean incidence age of HAS has been reported to be 63.5 years of age and more male patients have been observed, with a male to female ratio of 2.3:1 (7). The most common tumor site was identified to be the gastric antrum (7). The diagnosis of HAS is dependent on the pathological examination.

The formation of HAS may be regarded as consequence of embryogenesis, as the stomach and liver are derived from the foregut three to four months following fertilization. Gastric adenocarcinoma cells may be misdirected during differentiation and alternatively oriented towards hepatoid cells, the most characteristic of which are AFP-producing (8). AFP produced by HAS can be distinguished from AFP-producing gastric cancer (AFPPGC), another distinctive type of gastric cancer, as it exhibits a higher canavalin binding rate than that 
of AFPPGC (>90 vs. $<50 \%)(9)$. In a study by Ooi et al, the difference in canavalin binding was hypothesized to be due to the various origin cells of AFP. In AFPPGC, AFP was secreted by gastric adenocarcinoma cells, while it was secreted by hepatoid cells in cases of HAS (10).

As a rare subtype of gastric malignant tumors, HAS has been reported in individual cases from various therapeutic centers $(1,3,5,6,9)$. The elevated serum AFP level is regarded as a significant feature of HAS (11) and, among the previously reported HAS patients, $84.8 \%$ were observed to have elevated AFP levels ranging from $10-475000 \mathrm{ng} / \mathrm{ml}$ (12).

In a number of previously reported cases, the pathological features of HAS were as follows: i) HAS cells were large and polygonal with prominent nucleoli and abundant cytoplasm. The polygonal tumor cells were eosinophilous under hematoxylin and eosin staining, forming myeloid or cord structures, separated by sinusoidal capillaries $(3,14)$; ii) trabecular and intestinal-like structures may be identified in HAS cells and were defined as two pathological subtypes $(7,12,14)$. In these subtypes, hyaline particles may be observed, indicated by positive periodic acid-Schiff staining $(3,13)$; iii) steatosis and biliation were identified in a number of HAS cells $(11,12)$; iv) ultra microstructure of the HAS cells showed microvilli differentiation, originating from gastrointestinal epithelial cells $(3,8,13)$; and v) characteristic immunohistochemical staining, as reported in the literature, consisted of positive AFP expression in the majority of HAC patients $(91.6 \%)$ and positive CEA staining observed in $78.7 \%$ cases $(1,10,14)$. Additionally, cells were commonly observed to be positive for $\alpha 1$-antitrypsin and $\alpha 1$-antichymotrysin following immunohistochemical analysis $(13,14)$.

The clinical symptoms are not specific enough to accurately determine the diagnosis of HAS, as similar symptoms including nausea, loss of appetite and epigastric distress may be present in a variety of malignant tumors of the digestive system. Therefore, in gastric tumor cases with an elevated serum AFP level, the possibility of HAS must be considered. Preoperative computed tomography and $\beta$-ultrasound of the liver are routinely recommended for the exclusion of HCC, hepatic metastasis, teratoma and other diseases that may also produce AFP $(4,5,7)$. Histologically, the hepatoid structure identified in the gastric adenocarcinoma region may contribute significantly to the diagnosis (3-5,7). Immunohistochemical analysis may exhibit positive staining for epithelial membrane antigen and CEA, which may aid in the exclusion of gastric metastasis of $\operatorname{HCC}(4,8,10,11)$. New molecular markers, including palate, lung, and nasal epithelium clone protein and GATA4, may allow the differentiation of gastric hepatoid adenocarcinoma, HCC and CGC $(15,16)$.

Previous studies have reported that HAS frequently occurred with liver metastasis and exhibited a shorter interval between gastrectomy and liver metastasis than that of CGC, leading to a poorer prognosis $(17,18)$. As reported by Liu $e t a l$, the one-, three- and five-year survival rates of HAS were 30,13 and $9 \%$, respectively, compared with 95, 57 and 38\%, respectively in the CGC group (19). The poorer prognosis indicated a more aggressive biological behavior of HAS compared with that of CGC; the mechanism of this was proposed to be due to the immunosuppressive and protease-inhibitory properties, which may enhance the invasiveness of the tumor (20).
Due to the limited number of reported cases, there is currently a lack of understanding surrounding HAS. Therefore, further clinical studies and information are required for clinicians and pathologists to improve the diagnosis and treatment for this subtype of gastric cancer.

\section{References}

1. Ishikura H, Fukasawa Y, Ogasawara K, et al: An AFP-producing gastric carcinoma with features of hepatic differentiation: A case report. Cancer 56: 840-848, 1985.

2. Inoue M, Sano T, Kuchiba A, et al: Long-term results of gastrectomy for alpha-fetoprotein-producing gastric cancer. Br J Surg 97: 1056-1061, 2010.

3. Zuo W, Dai G, Liu L and Zuo H: Pathological character and clinical analysis of the hepatoid adenocarcinoma of stomach: A report of 11 cases. Chin Clin Ocol 14: 923-926, 2009 (In Chinese).

4. Chang YC, Nagasue N, Abe S, et al: Comparison between the clinicopathologic features of AFP-positive and AFP-negative gastric cancers. Am J Gastroenterol 87: 321-325, 1992.

5. Baek SK, Han SW, Oh DY, et al: Clinicopathologic characteristics and treatment outcomes of hepatoid adenocarcinoma of the stomach, a rare but unique subtype of gastric cancer. BMC Gastroenterol 11: 56, 2011.

6. Ye M, Tao F, Liu F and Sun AJ: Hepatoid adenocarcinoma of the stomach: a report of three cases. World J Gastroenterol 19: 4437-4442, 2013

7. Su JS, Chen YT, Wang RC, et al: Clinicopathological characteristics in the differential diagnosis of hepatoid adenocarcinoma: a literature review. World J Gastroenterol 19: 321-327, 2013.

8. Gitlin D, Perricelli A and Gitlin GM: Synthesis of -fetoprotein by liver, yolk sac, and gastrointestinal tract of the human conceptus. Cancer Res 32, 979-982, 1972.

9. Bakir T, Aliyazicioglu Y, Bektas A, et al: Hepatoid adenocarcinoma of the stomach: report of five cases and review of literature. Acta Gastroenterol Belg 69: 330-337, 2006.

10. Ooi A, Nakanishi I, Sakamoto N, et al: Alpha-fetoprotein (AFP)-producing gastric carcinoma. Is it hepatoid differentiation? Cancer 65: 1741-1747, 1990.

11. Kinjo T, Taniguchi H, Kushima R, et al: Histologic and immunohistochemical analyses of $\alpha$-fetoprotein-producing cancer of the stomach. Am J Surg Pathol 36: 56-65, 2012.

12. Bruix J and Sherman M; Practice Guidelines Committee, American Association for the Study of Liver Diseases: Management of hepatocellular carcinoma. Hepatology 42: 1208-1236, 2005.

13. Ishikura H, Kirimoto K, Shamoto M, et al: Hepatoid adenocarcinomas of the stomach. An analysis of seven cases. Cancer 58: 119-126, 1986.

14. Plaza JA, Vitellas K and Frankel WL: Hepatoid adenocarcinoma of the stomach. Ann Diagn Pathol 8: 137-141, 2004.

15. Sentani K, Oue N, Sakamoto N, et al: Gene expression profiling with microarray and SAGE identifies PLUNC as a marker for hepatoid adenocarcinoma of the stomach. Mod Pathol 21: 464-475, 2008.

16. Yamamura N and Kishimoto T: Epigenetic regulation of GATA4 expression by histone modification in AFP-producing gastric adenocarcinoma. Exp Mol Pathol 93: 35-39, 2012.

17. Nagai E, Ueyama T, Yao T, et al: Hepatoid adenocarcinoma of the stomach: A clinicopathologic and immunohistochemical analysis. Cancer 72: 1827-1835, 1993.

18. Liu X, Cheng Y, Sheng W, et al: Analysis of clinicopathologic features and prognostic factors in hepatoid adenocarcinoma of the stomach. Am J Surg Pathol 34: 1465-1471, 2010.

19. Liu X, Sheng W and Wang Y: An analysis of clinicopathological features and prognosis by comparing hepatoid adenocarcinoma of the stomach with AFP-producing gastric cancer. J Surg Oncol 106: 299-303, 2012.

20. Kono K, Amemiya H, Sekikawa T, et al: Clinicopathologic features of gastric cancers producing alpha-fetoprotein. Dig Surg 19: $359-365,2002$. 